\title{
Multicenter phase II study of infusional 5-fluorouracil (5-FU), leucovorin, and oxaliplatin, plus biweekly cetuximab as first-line treatment in patients with metastatic colorectal cancer (CELINE trial)
}

\author{
MASANORI KOTAKE ${ }^{1 *}$, TORU AOYAMA $^{2 *}$, YOSHINORI MUNEMOTO $^{3}$, KENJI DODEN $^{4}$, MASATO KATAOKA $^{5}$, \\ KENJI KOBAYASHI ${ }^{6}$, GENICHI NISHIMURA ${ }^{7}$, HIDEHITO FUJITA ${ }^{8}$, KEISHI NAKAMURA ${ }^{9}$, AKIRA TAKEHARA ${ }^{10}$, \\ CHIHIRO TANAKA $^{11}$, JUNICHI SAKAMOTO ${ }^{12}$, NAOKI NAGATA ${ }^{13}$, KOJI OBA ${ }^{14}$ and KEN KONDO ${ }^{5}$ \\ ${ }^{1}$ Department of Surgery, Kouseiren Takaoka Hospital, Takaoka, Toyama 933-8555; \\ ${ }^{2}$ Department of Gastrointestinal Surgery, Kanagawa Cancer Center, Yokohama, Kanagawa 241-8515; \\ ${ }^{3}$ Department of Surgery, Fukui Saiseikai Hospital, Fukui 918-8503; ${ }^{4}$ Department of Surgery, Fukui Prefectural Hospital, \\ Fukui 910-8526; ${ }^{5}$ Department of Surgery, National Hospital Organization (NHO) Nagoya Medical Center, Nagoya, \\ Aichi 460-0001; ${ }^{6}$ Department of Surgery, Matsunami General Hospital, Gifu 501-6062; ${ }^{7}$ Department of Surgery, \\ Japanese Red Cross Kanazawa Hospital, Kanazawa 921-8162; ${ }^{8}$ Department of Surgery, \\ Kanazawa Medical University, Uchinada, Kanazawa 920-0293; ${ }^{9}$ Department of Surgery, Kanazawa University Hospital, \\ Kanazawa 920-8641; ${ }^{10}$ Department of Surgery, Japanese Red Cross Toyama Hospital, Toyama 930-0859; \\ ${ }^{11}$ Department of Surgery, Gifu Prefectural General Medical Center, Gifu 500-8717; ${ }^{12}$ Department of Surgery, \\ Tokai Central Hospital, Kakamigahara, Gifu 504-8601; ${ }^{13}$ Department of Surgery, Kitakyushu General Hospital, \\ Kitakyushu, Fukuoka 802-8517; ${ }^{14}$ Department of Biostatistics, The University of Tokyo, Tokyo 113-8654, Japan
}

Received November 19, 2015; Accepted October 21, 2016

DOI: $10.3892 / 01.2016 .5505$

\begin{abstract}
The current phase II study investigated the efficacy and safety of biweekly cetuximab combined with standard oxaliplatin-based chemotherapy [infusional 5-fluorouracil (5-FU), leucovorin, and oxaliplatin (FOLFOX-6)] in the first-line treatment of KRAS wild-type metastatic colorectal cancer (mCRC). Sixty patients with a median age of 64 years (range, 38-82 years) received a biweekly intravenous infusion of cetuximab $\left(500 \mathrm{mg} / \mathrm{m}^{2}\right.$ on day 1) followed by FOLFOX-6 (2-hour oxaliplatin $85 \mathrm{mg} / \mathrm{m}^{2}$ infusion on day 1 in tandem with a 2-h leucovorin $200 \mathrm{mg} / \mathrm{m}^{2}$ infusion on days 1 and 2 , and 5 -FU as a $400 \mathrm{mg} / \mathrm{m}^{2}$ bolus followed by a 46 -hour $2,400 \mathrm{mg} / \mathrm{m}^{2}$ infusion on days 1-3). Patient response rate was $70 \%$, with $95 \%$ disease control rates. The median progression-free survival was 13.8 months. Thirteen patients $(21.7 \%)$ were able to undergo resection of previously unresectable metastases, with the aim
\end{abstract}

Correspondence to: Dr Toru Aoyama, Department of Gastrointestinal Surgery, Kanagawa Cancer Center, 2-3-2 Nakao, Asahi-ku, Yokohama, Kanagawa 241-8515, Japan

E-mail: aoyamat@kcch.jp

${ }^{*}$ Contributed equally

Key words: Cetuximab, FOLFOX, colorectal cancer, mCRC of curing them. The median follow-up was 22.7 months, and median overall survival was 31.0 months. Cetuximab did not increase FOLFOX-6 toxicity and was generally well tolerated. The results of the current study demonstrate that the combination of biweekly cetuximab with FOLFOX-6 was well tolerated and had a manageable safety profile for the first-line treatment of KRAS wild-type metastatic colorectal cancer. Efficacy was comparable to other treatment regimens. The results support the administration of biweekly cetuximab in combination with FOLFOX-6, which may be more convenient and provide treatment flexibility in this setting for patients with metastatic colorectal cancers.

\section{Introduction}

Colorectal cancer is the third most common cancer and one of the most frequent causes of cancer-associated mortality worldwide (1). The main method of treating it is by complete resection, however, metastatic colorectal cancer (mCRC) cannot be treated by surgical resection alone, and the prognosis of such patients is poor. Although there have been substantial advances in mCRC treatment, the median survival rate of patients with mCRC remains $<3$ years and $<5 \%$ of patients survive $>5$ years following disease onset (2-4).

The introduction of 5-fluorouracil (5-FU), oxaliplatin and irinotecan (FOLFOX), as a therapeutic strategy has improved $\mathrm{mCRC}$ treatment $(3,5,6)$. The addition of targeted therapies to conventional mCRC chemotherapy regimens has 
improved treatment efficacy further. Cetuximab is a chimeric human mouse monoclonal antibody of the immunoglobulin G1 (IgG1) subclass that targets the epidermal growth factor receptor (EGFR). Cetuximab binds to human EGFR with high affinity and inhibits the multiplication of various EGFR-positive cancer cell lines in vitro in a concentration-dependent manner (6,7). Previous studies have indicated that cetuximab is an effective treatment of $\mathrm{mCRC}$ when administered either as monotherapy or in combination with other chemotherapeutic agents $(8,9)$. Furthermore, two randomized clinical trials have demonstrated the clinical efficacy of adding weekly cetuximab to irinotecan- or oxaliplatin-based chemotherapy regimens to treat patients with $\mathrm{mCRC}$ who do not exhibit the mutated KRAS proto-oncogene, GTPase (KRAS wild-type mCRC) $(10,11)$.

Cetuximab is approved in a number of countries for clinical use in patients with KRAS wildtype mCRC. The current dosing regimen of cetuximab, both as a monotherapy and in combination with chemotherapy, consists of an initial intravenous infusion of $400 \mathrm{mg} / \mathrm{m}^{2}$ with subsequent weekly doses of $250 \mathrm{mg} / \mathrm{m}^{2}$. Changing the cetuximab treatment schedule to biweekly administration would improve convenience (12). These benefits would be even greater for $\mathrm{mCRC}$ treatment regimens as the standard chemotherapy regimens used in combination with cetuximab in KRAS wild-type mCRC; FOLFOX (4 or 6), or irinotecan plus 5-FU infusion and leucovorin (FOLFIRI), are already administered every two weeks. Moreover, the synchronous administration of cetuximab and chemotherapy would improve patient quality of life, as hospital visits would be less frequent, and patients would not experience the unpleasant side effects associated with chemotherapy treatment as often.

The present phase II study aimed to evaluate the efficacy and safety of the biweekly administration of cetuximab in combination with FOLFOX-6 as a first-line treatment of KRAS wild-type mCRC. Changing to a biweekly regimen may be more convenient and markedly improve patient quality of life.

\section{Patients and methods}

Study design. The present study was a multicenter, open-label, prospective phase II study investigating the safety and efficacy of cetuximab combined with FOLFOX as a first-line treatment of mCRC. The study data and informed consent were obtained in accordance with the Declaration of Helsinki, and the study protocol was approved by the Ethics Review Board of each institution involved.

Inclusion and exclusion criteria. Patients were eligible if they were $\geq 20$ years of age; had histologically confirmed, nonresectable, EGFR-expressing mCRC with at least one radiologically measurable lesion; an Eastern Cooperative Oncology Group performance status $<1$; and adequate hepatic, renal, and bone marrow function (white blood cell count: $3,000-12,000 / \mathrm{mm}^{3}$, neutrophil count $\geq 1,500 / \mathrm{mm}^{3}$, platelet count $\geq 100,000 / \mathrm{mm}^{3}$, aspartate aminotransferase and alanine aminotransferase $\leq 100 \mathrm{U} / 1$, total bilirubin $<1.5 \mathrm{mg} / \mathrm{dl}$, creatinine $<1.5 \mathrm{mg} / \mathrm{dl}$, and normal electrocardiogram). Patients were ineligible if they had a history of previous exposure to EGFR-targeted therapy, previous chemotherapy for $\mathrm{mCRC}$, or uncontrolled severe organ/metabolic dysfunction.

Study treatment. Patients received a biweekly intravenous infusion of cetuximab $\left(500 \mathrm{mg} / \mathrm{m}^{2}\right.$ on day 1$)$ followed by FOLFOX-6 (2-h oxaliplatin $85 \mathrm{mg} / \mathrm{m}^{2}$ infusion on day 1 in tandem with a $2-\mathrm{h}$ leucovorin $200 \mathrm{mg} / \mathrm{m}^{2}$ infusion on days 1 and 2 , and $5-\mathrm{FU}$ as a $400 \mathrm{mg} / \mathrm{m}^{2}$ bolus followed by a $46-\mathrm{h}$ $2,400 \mathrm{mg} / \mathrm{m}^{2}$ infusion on days $1-3$ ). Cetuximab was administered over $2 \mathrm{~h}$ in the first cycle, over $1.5 \mathrm{~h}$ in the second cycle and over $1 \mathrm{~h}$ thereafter. Appropriate prophylactic medication was administered before each cetuximab treatment to prevent acute hypersensitivity reaction. Protocol dose modifications were permitted in the event of predefined toxic effects related to chemotherapy or cetuximab. In the event of unacceptable toxicity due to 5-FU/leucovorin, oxaliplatin, or cetuximab, the trial was discontinued, and the patient was transferred to an alternate treatment regimen. However, protocol modifications did not allow the maintenance of oxaliplatin as a monotherapy or in combination with cetuximab. Treatment was continued until disease progression, the onset of unacceptable toxic effects, patient/physician's decision to discontinue, or surgery for metastases.

KRAS and BRAF mutations. DNA was extracted from formalin-fixed, paraffin-embedded tumor tissues. Mutations of KRAS at codons 12, 13, and 61 and BRAF mutations at codon 600 were detected by direct sequencing, as described previously $(13,14)$.

Statistical analysis. The primary endpoint was the best overall response. The response evaluation criteria in solid tumors (RECIST) 1.1 criteria were applied to assess and confirm overall response (15). A radiological assessment of the response was performed at the end of the fourth and sixth cycles and then every six cycles until the end of the study. If progressive disease (PD) had not been observed, the radiological assessment was subsequently performed every 12 weeks, as per routine clinical practice. Secondary endpoints were: Progression-free survival (PFS), overall survival (OS), and the safety of the combination therapy. Computed tomography or magnetic resonance imaging were performed at baseline, every 8 weeks during the first 6 months of the study, and every 12 weeks thereafter, until disease progression. Adverse events were recorded throughout the study period and were graded according to the Common Toxicity Criteria of the National Cancer Institute ver. 3.0 (16).

All summary statistics on time-to-event variables were calculated according to Kaplan-Meier methods. The $95 \%$ confidence intervals (CI) for median time-to event and the time-specific incidence rate were constructed using Greenwood's formula, and Brookmeyer and Crowley's method, respectively. The PFS of patients without disease progression before the end of the study was calculated from the last on-study tumor assessment date at which the patient was considered to be progression-free. PFS was defined as the number of days between enrollment and the first on-study assessment of PD; OS was defined as the number of days between enrollment and any cause of death or the last follow-up. Patients without disease progression who 
discontinued the study for any reason were restricted to the last on-study tumor assessment date.

A sample size of 55 patients was deemed as the smallest sample size from which accurate conclusions could be formed and was calculated to detect a $95 \%$ CI for the overall response rate (ORR), with a half-width of $13 \%$, assuming an estimated rate of $60 \%$ calculated from previous studies. Therefore, the target sample size was set at 60 patients, with an anticipated $10 \%$ patient loss to follow-up.

\section{Results}

Patient characteristics. From October 2011 to February 2013, 33 institutions collaborated with the CELINE study, and 65 patients were registered from 21 institutions. Five patients were excluded from the study: Three patients had EGFR mutations, one patient had no information regarding EGFR status, and one patient was unable to begin treatment. In the end, 60 patients were deemed eligible for the present study, and regarded as ready-to-treat. A safety analysis was carried out on all patients following administration of $\geq 1$ dose of any component of the study treatment. Patient clinical characteristics at baseline were documented and are presented in Table I. The median age of the patients was 64 years (range, 38-82 years). There were 34 patients $(56.7 \%)$ with metastatic lesions in one organ, and 26 patients $(43.3 \%)$ with metastatic lesions in $>1$ organ. Hepatic metastases were the most common lesion, with such lesions detected in 50 patients $(83.3 \%)$. The median duration of follow-up was 22.7 months.

Efficacy. Efficacy data are summarized in Table II. The best confirmed ORR was 70\% (95\% CI, 56.8-81.2\%; 2 complete and 40 partial responses). Moreover, the best confirmed ORR was $72 \%$ in patients with hepatic metastases $(n=50,95 \%$ CI, $57.5-83.8 \%), 73.3 \%$ in patients with lung metastases $(n=15,95 \%$ CI, 44.9-92.2\%), and $63.2 \%$ in patients with lymphatic metastases $(n=19,95 \%$ CI, 38.4-83.7\%); there were no observable differences in the ORR between different metastatic organs. There were 15 patients (25\%) with stable disease, and objective disease progression was observed in 1 patient. Therefore, the disease control rate (DCR) was 95\% (95\% CI, 86.1-99.0\%), demonstrating treatment efficacy. In the 42 patients exhibiting partial or complete response, the median time to onset of response was 2.1 months.

After a median follow-up of 22.7 months, the median PFS was 13.8 months (95\% CI, 11.7-17.1; Fig. 1), and the median OS was 31.0 months (95\% CI, 21.6-NA; Fig. 2).

There were 13 patients $(21.7 \%)$ who underwent surgery for liver metastases, and complete surgical resection was observed in 10 patients. The median PFS following surgical resection of the metastases was 14.9 months. Median OS was not evaluable. Nine of the 26 patients receiving second-line chemotherapy continued with cetuximab-containing regimens following surgery.

Treatment compliance and safety. The treatment exposure is summarized in Table III. The median course of study treatment was 10.5 months (range, 2.0-52.0). Patients achieving relative dose intensity $\geq 80 \%$ for each drug is presented (Table III). Adverse events (AEs) of any grade occurred in
Table I. Patient characteristics.

Percentage of

Characteristics

No. patients patients $(\%)$

\begin{tabular}{lcr}
\hline Gender & & \\
Male & 47 & 78.3 \\
Female & 13 & 21.7 \\
Age (years) & & \\
Median & 64 & N/A \\
Range & $38-82$ & N/A \\
ECOG performance status & & \\
0 & 51 & 85.0 \\
1 & 9 & 15.0 \\
Cancer location & & \\
Colon & 30 & 50.0 \\
Rectum & 30 & 50.0 \\
Previous adjuvant therapy & & \\
Chemotherapy & 4 & 6.7 \\
Non & 56 & 93.3 \\
Number of organs with metastases & & \\
1 & 34 & 56.7 \\
2 & 14 & 23.3 \\
$\geq 3$ & 12 & 20.0
\end{tabular}

mCRC, metastatic colorectal cancer; EGFR, epidermal growth factor receptor; ECOG, Eastern Cooperative Oncology Group; Intention to treat population, $\mathrm{n}=60$.

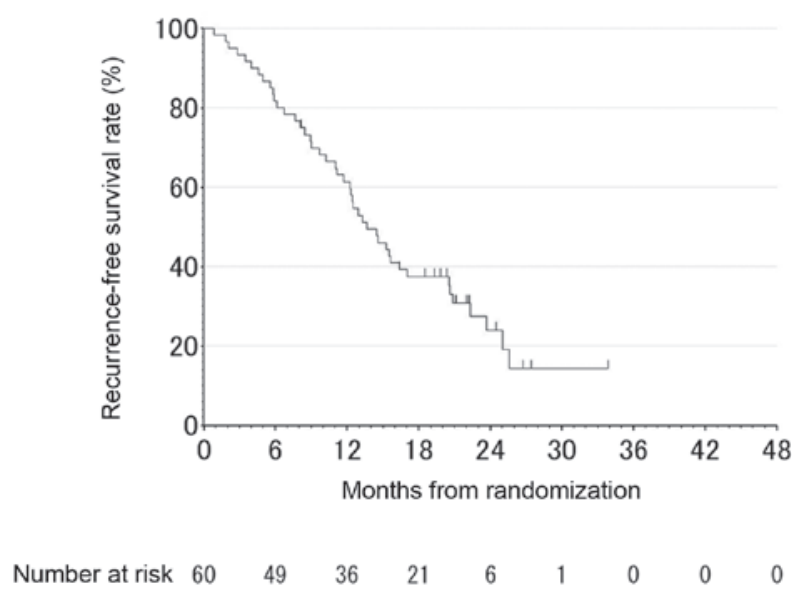

Figure 1. Progression-free survival.

all patients (100\%), and grade 3 or 4 AEs (classed as severe or life-threatening) were observed in $66.7 \%$ (40/60 patients). Patient AEs are summarized in Table IV. The most common grade 3 or 4 AEs were leutropenia (41.7\%) and paronychia $(18.3 \%)$. Out of all the patients presenting with grade 3 or 4 AE, $8.3 \%$ presented with an acne-like rash and one patient presented with infusion-related reactions (this special event category included the preferred terms 'infusion-related reaction' and 'pyrexia'). 
Table II. Efficacy data.

\begin{tabular}{lcc}
\hline Parameter & No. patients $(\%)(\mathrm{n}=60)$ & $95 \%$ confidence interval \\
\hline Best overall response rate & $2(3.3)$ & \\
Complete response (CR) & $40(66.7)$ \\
Partial response (PR) & $16(26.7)$ & $56.8-81.2$ \\
Stable disease (SD) & $1(1.7)$ & $88.5-99.6$ \\
Progressive disease (PD) & $1(1.7)$ & $11.2-16.4$ \\
Not assessable & $42(70.0)$ & \\
Best overall response rate (CR+PR) & $58(96.7)$ & $47.8-72.4$ \\
Disease control rate (CR+PR+SD) & 13.7 & $21.6-\mathrm{N} / \mathrm{A}$ \\
Median progression free survival, months & 46 & $65.2-86.6$ \\
Progression events & 14 & $38.8-69.1$ \\
Censored & 61.4 & \\
Progression free rate at 12 months & 31.0 & \\
Median overall survival, months & 24 & \\
Deaths & 36 & \\
Censored & 78.1 & \\
1-year survival rate & 55.3 & \\
2-year survival rate & 20 & \\
Surgery for metastatic disease & 13 & \\
Liver surgery & 2 & \\
Lung surgery & 7 & \\
Other organ surgery & & \\
Results of surgery & 13 & \\
No residual tumor after the resection & 3 & \\
Microscopic (R1) residual lesion & & \\
\hline & &
\end{tabular}

Table III. Treatment exposure.

\begin{tabular}{lccc}
\hline Treatment parameter & Cetuximab & Oxaliplatin & Fluorouracil \\
\hline Median dose-intensity $\left(\mathrm{mg} / \mathrm{m}^{2} /\right.$ week) & 227 & 32 & 1010 \\
Median dose-intensity $\left(\mathrm{mg} / \mathrm{m}^{2} / 2\right.$ weeks) & 430 & 61 & 1917 \\
Relative dose-intensity $(\%)$ & & & \\
Median & 86.3 & 72.5 & 79.9 \\
$\geq 90$ & 38.3 & 16.7 & 31.7 \\
$\geq 80$ to $<90$ & 31.7 & 15.0 & 18.3 \\
$\geq 60$ to $<80$ & 21.7 & 35.0 & 36.7 \\
$<60$ & 8.3 & 33.3 & 13.3 \\
\hline
\end{tabular}

\section{Discussion}

The current study aimed to evaluate the response rate of patients with KRAS wild-type mCRC to the first-line treatment of FOLFOX-6 combined with biweekly cetuximab. The results indicated that the efficacy and safety of this method of treatment is similar to those observed in previous phase II studies that evaluated the biweekly administered cetuximab regimen and reported the standard weekly cetuximab dosing regimen as a first-line treatment of KRAS wild-type $\operatorname{mCRC}(17,18)$.
The confirmed best ORR was 70\% (95\% CI, 56.8-81.2\%), and similar ORR was observed regardless of the metastatic organ site. The ORR results obtained from the present study are similar to a previous phase II study evaluating the efficacy of biweekly administration of cetuximab in combination with FOLFOX-4. Furthermore, two other phase II trials have evaluated the efficacy and safety of a biweekly administration of cetuximab in combination with FOLFOX-4 to treat mCRC. One study was performed by the Central European Cooperative Oncology Group (CECOG) (19). This randomized phase II study investigated the efficacy and safety of chemotherapy 
Table IV. Relevant adverse events in $\geq 10 \%$ of patients (highest grade/patient).

\begin{tabular}{|c|c|c|c|c|}
\hline \multirow[b]{2}{*}{ Adverse event } & \multicolumn{2}{|c|}{ Grade III or IV } & \multicolumn{2}{|c|}{ All grade } \\
\hline & No. patients & Percentage, $\%$ & No. patients & Percentage, $\%$ \\
\hline \multicolumn{5}{|l|}{ Hematological } \\
\hline White blood cell decrease & 6 & 10.0 & 38 & 63.3 \\
\hline Leukopenia & 25 & 41.7 & 32 & 53.3 \\
\hline Anemia & 2 & 3.3 & 45 & 75.0 \\
\hline Thrombocytopenia & 0 & 0 & 38 & 63.3 \\
\hline \multicolumn{5}{|l|}{ Non hematological } \\
\hline Elevated AST/ALT & 0 & 0 & 41 & 68.3 \\
\hline Elevated serum bilrubin & 0 & 0 & 5 & 8.3 \\
\hline Elevated creatine & 0 & 0 & 14 & 23.3 \\
\hline Stomatitis & 2 & 3.3 & 27 & 45.0 \\
\hline Nausea/vomiting & 2 & 3.3 & 27 & 45.0 \\
\hline Diarrhea & 0 & 0 & 16 & 26.7 \\
\hline Rash & 5 & 8.3 & 53 & 88.3 \\
\hline Paronychia & 11 & 18.3 & 45 & 75.0 \\
\hline Anorexia & 2 & 3.3 & 31 & 51.7 \\
\hline Fatigue & 1 & 1.7 & 28 & 46.6 \\
\hline Infusion related reaction & 1 & 1.7 & 4 & 6.7 \\
\hline Paresthesia & 5 & 8.3 & 53 & 88.3 \\
\hline
\end{tabular}

AST/ALT, aspartate transaminase/alanine transanimase.

plus cetuximab administered every second week as a first-line treatment for KRAS wild-type mCRC. In this trial, the ORR was $62 \%(95 \% \mathrm{CI}, 51-73 \%)$ in the biweekly cetuximab arm. Another phase II, open-label clinical trial (the OPTIMIX-ACROSS study), evaluated biweekly cetuximab in combination with FOLFOX-4 as first-line treatment for KRAS wild-type mCRC (17). This phase II study was carried out in 15 Spanish centers and the ORR was 60.6\% (95\% CI, $50.3-70.3 \%)$ in the biweekly cetuximab arm. In addition, the confirmed best ORR achieved in the current study was similar to a previous phase II study, Oxaliplatin and Cetuximab in First-Line Treatment of Metastatic Colorectal Cancer (OPUS), investigating the efficacy of a weekly administration of cetuximab in combination with FOLFOX-4. The ORR was reported as $57 \%$ (95\% CI, 46-68\%) in the KRAS wild-type population and $60 \%(95 \% \mathrm{CI}, 48-71 \%)$ in the KRAS/BRAF wild-type population in patients receiving weekly infeusions of cetuximab and FOLFOX-4 (11).

The median PFS was 13.8 months (95\% CI, 11.7-17.1 months) in the present study. This was similar to the median PFS reported in the OPTIMIX-ACROSS study and the CECOG trial, which were 10.1 months and 9.2 months respectively $(17,19)$. Furthermore, it was slightly longer than the range of 8.4-9.1 months previously reported for the standard weekly cetuximab administration in combination with other oxaliplatin-based chemotherapy regimens in patients with KRAS wild-type mCRC (18).

The feasibility of a biweekly cetuximab administration schedule was previously investigated in a two-part phase I dose escalation study (20). This study demonstrated that cetuximab

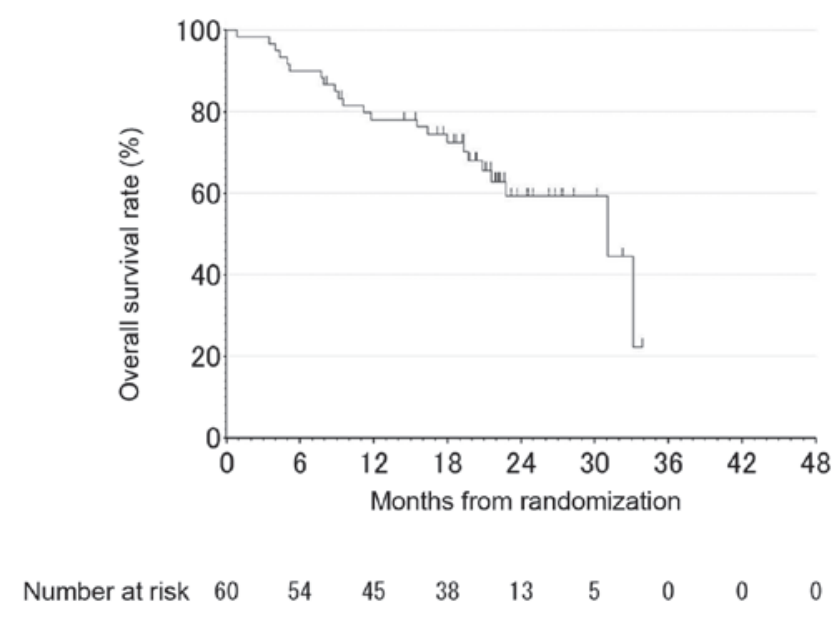

Figure 2. Overall survival.

may be safely administered as a single agent or in combination with FOLFIRI at doses of $400-700 \mathrm{mg} / \mathrm{m}^{2}$ in a biweekly schedule, and $500 \mathrm{mg} / \mathrm{m}^{2}$ was established as the recommended dose according to the pharmacokinetic exposure data. Therefore, in the present study, the patients received a biweekly intravenous infusion of cetuximab $\left(500 \mathrm{mg} / \mathrm{m}^{2}\right.$ on day 1) followed by FOLFOX-6. Most patients $(66.7 \%)$ experienced severe AEs (grade 3 or 4), however, this is normal compared with patients undergoing other treatment regimes, demonstrating that the combination of cetuximab and FOLFOX was generally well tolerated. There was no evidence in the current study suggesting that cetuximab increased the frequency or 
severity of characteristic toxicities associated with FOLFOX treatment. Indeed, the proportion of patients with grade 3 or 4 AEs observed in the current study was similar to those in previous studies: $77.8 \%$ in the OPTIMIX-ACROSS study and $71 \%$ in the biweekly arm of the CECOG trial $(17,19)$. Moreover, typical and manageable EGFR-inhibitor-related AEs, such as a rash or acne-like rash, were reported at grades 3 or 4 for $8.3 \%$ of the patients, similar to previous studies.

The frequency of bone marrow suppression recorded was markedly higher than that of previous studies. Incidences of leukopenia, neutropenia, anemia, and thrombocytopenia were $63.3,53.3,75.0$, and $63.3 \%$, respectively, in the present study, compared with $15.2,49.5,11.1$, and $23.2 \%$ in the OPTIMIX-ACROSS study (17). However, the frequency of severe bone marrow suppression (grade 3 or 4) requiring dose-reduction or treatment delay was similar. The incidences of leukopenia, neutropenia, anemia, and thrombocytopenia were $10.0,41.7,3.3$, and $0 \%$, respectively, in the present study, compared with $1.0,32.3,2.0$, and $2.0 \%$, respectively in the OPTIMIX-ACROSS Study. Moreover, the Multicenter Phase II study of FOLFOX or biweekly XELOX and Erbitux (cetuximab) as first-line therapy in patients with wild-type $K R A S / B R A F$ metastatic colorectal cancer (The FLEET study), documented a similar toxicity: The incidences of grade 3 or 4 leukopenia, neutropenia, and thrombocytopenia were 10.8, 29.7, and 5.4\% in patients treated with FOLFOX-6 and cetuximab (20).

The present study demonstrated the equivalent efficacy and safety of biweekly cetuximab in combination with FOLFOX-6 for patients with KRAS wild-type mCRC compared with the current standard first-line treatment. There are numerous advantages of administering cetuximab biweekly instead of weekly (14). Synchronous cetuximab and chemotherapy administration is much more convenient for patients with mCRC. A reduction in the frequency of hospital visits may improve patient quality of life. Furthermore, the biweekly combination regimen may simplify treatment administration for health care workers and be more economical; a simplified administration schedule may decrease the costs associated with frequent cetuximab administration as well as frequent hospital visits. Thus, biweekly-administered cetuximab in combination with FOLFOX-6 may benefit governments, patients, physicians, and other health care workers involved in treating mCRC.

One important limitation of the present study is the lack of information regarding tumor makers. Tumor makers are an important measurement of treatment efficacy, however; tumor marker measurement was not included as an endpoint. Instead, the primary endpoint of the present study was the overall response rate, and secondary endpoints were progression-free survival, overall survival, disease control rate, the incidence of adverse events, and response rate at each metastatic site. Therefore further studies collecting tumor marker measurements and taking them into consideration are required.

In conclusion, the present study demonstrated that biweekly administration of cetuximab in combination with FOLFOX-6 has a feasible and manageable safety profile for the first-line treatment of KRAS wild-type mCRC. The results support a biweekly cetuximab regimen as a novel therapeutic strategy to treat KRAS wild-type mCRC, which may be more convenient for patients and provide more flexibility for those administering the treatment.

\section{Acknowledgements}

The present study was supported, in part, by the non-profit organization Epidemiological \& Clinical Research Information Network (ECRIN). The authors are especially grateful to Ms. Mai Hatta and Ms. Chikako Ikeda for their excellent contributions as clinical research coordinators of this study.

\section{References}

1. Ferlay J, Soerjomataram I, Dikshit R, Eser S, Mathers C, Rebelo M, Parkin DM, Forman D and Bray F: Cancer incidence and mortality worldwide: Sources, methods and major patterns in GLOBOCAN 2012. Int J Cancer 136: E359-E386, 2014.

2. Douillard JY, Cunningham D, Roth AD, Navarro M, James RD, Karasek P, Jandik P, Iveson T, Carmichael J, Alakl M, et al: Irinotecan combined with fluorouracil compared with fluorouracil alone as first-line treatment for metastatic colorectal cancer: A multicentre randomised trial. Lancet 355: 1041-1047, 2000.

3. Saltz LB, Cox JV, Blanke C, Rosen LS, Fehrenbacher L, Moore MJ, Maroun JA, Ackland SP, Locker PK, Pirotta N, et al: Irinotecan plus fluorouracil and leucovorin for metastatic colorectal cancer. Irinotecan Study Group. N Engl J Med 343: 905-914, 2000.

4. Köhne CH, van Cutsem E, Wils J, Bokemeyer C, El-Serafi M, Lutz MP, Lorenz M, Reichardt P, Rückle-Lanz H, Frickhofen N, et al: Phase III study of weekly high-dose infusional fluorouracil plus folinic acid with or without irinotecan in patients with metastatic colorectal cancer: European Organisation for Research and Treatment of Cancer Gastrointestinal Group Study 40986. J Clin Oncol 23: 4856-4865, 2005.

5. de Gramont A, Figer A, Seymour M, Homerin M, Hmissi A, Cassidy J, Boni C, Cortes-Funes H, Cervantes A, Freyer G, et al: Leucovorin and fluorouracil with or without oxaliplatin as first-line treatment in advanced colorectal cancer. J Clin Oncol 18: 2938-2947, 2000.

6. Sunada H, Magun BE, Mendelsohn J and MacLeod CL: Monoclonal antibody against epidermal growth factor receptor is internalized without stimulating receptor phosphorylation. Proc Natl Acad Sci USA 83: 3825-3829, 1986.

7. Li S, Schmitz KR, Jeffrey PD, Wiltzius JJ, Kussie P and Ferguson KM: Structural basis for inhibition of the epidermal growth factor receptor by cetuximab. Cancer Cell 7: 301-311, 2005.

8. Cunningham D, Humblet Y, Siena S, Khayat D, Bleiberg H, Santoro A, Bets D, Mueser M, Harstrick A, Verslype C, et al: Cetuximab monotherapy and cetuximab plus irinotecan in irinotecan-refractory metastatic colorectal cancer. N Engl J Med 351: 337-345, 2004.

9. Goldstein NS and Armin M: Epidermal growth factor receptor immunohistochemical reactivity in patients with American Joint Committee on Cancer Stage IV colon adenocarcinoma: Implications for a standardized scoring system. Cancer 92: 1331-1346, 2001.

10. Van Cutsem E, Köhne CH, Láng I, Folprecht G, Nowacki MP, Cascinu S, Shchepotin I, Maurel J, Cunningham D, Tejpar S, et al: Cetuximab plus irinotecan, fluorouracil, and leucovorin as first-line treatment for metastatic colorectal cancer: Updated analysis of overall survival according to tumor KRAS and BRAF mutation status. J Clin Oncol 29: 2011-2019, 2011.

11. Bokemeyer C, Bondarenko I, Hartmann JT, de Braud F, Schuch G, Zubel A, Celik I, Schlichting M and Koralewski P: Efficacy according to biomarker status of cetuximab plus FOLFOX-4 as first-line treatment for metastatic colorectal cancer: The OPUS study. Ann Oncol 22: 1535-1546, 2011.

12. Tabernero J, Pfeiffer P and Cervantes A: Administration of cetuximab every 2 weeks in the treatment of metastatic colorectal cancer: An effective, more convenient alternative to weekly administration? Oncologist 13: 113-119, 2008.

13. Okayama N, Nishioka M, Hazama S, Sakai K, Suehiro Y, Maekawa M, Sakamoto J, Iwamoto S, Kato T, Mishima H, et al: The importance of evaluation of DNA amplificability in KRAS mutation testing with dideoxy sequencing using formalin-fixed and paraffin-embedded colorectal cancer tissues. Jpn J Clin Oncol 41: 165-171, 2011. 
14. Iwamoto S, Hazama S, Kato T, Miyake Y, Fukunaga M, Matsuda C, Bando H, Sakamoto J, Oba K, Mishima H, et al: Multicenter phase II study of second-line cetuximab plus folinic acid/5-fluorouracil/irinotecan (FOLFIRI) in KRAS wild-type metastatic colorectal cancer: The FLIER study. Anticancer Res 34: 1967-1973, 2014.

15. Eisenhauer EA, Therasse P, Bogaerts J, Schwartz LH, Sargent D, Ford R, Dancey J, Arbuck S, Gwyther S, Mooney M, et al: New response evaluation criteria in solid tumours: Revised RECIST guideline (version 1.1). Eur J Cancer 45: 228-247, 2009.

16. NationalCancer and Institute CancerTherapyEvaluation Program:Common Terminology Criteria for Adverse Events v3.0 (CTCAE) https://ctep.cancer.gov/protocolDevelopment/electronic_applications/docs/ctcaev3.pdf

17. Fernandez-Plana J, Pericay C, Quintero G, Alonso V, Salud A, Mendez M, Salgado M, Saigi E and Cirera L; ACROSS Study Group: Biweekly cetuximab in combination with FOLFOX-4 in the first-line treatment of wild-type KRAS metastatic colorectal cancer: Final results of a phase I. BMC Cancer 14: 865, 2014.
18. Soda H, Maeda H, Hasegawa J, Takahashi T, Hazama S, Fukunaga M, Kono E, Kotaka M, Sakamoto J, Nagata N, et al: Multicenter Phase II study of FOLFOX or biweekly XELOX and Erbitux (cetuximab) as first-line therapy in patients with wild-type KRAS/BRAF metastatic colorectal cancer: The FLEET study. BMC Cancer 15: 695, 2015.

19. Brodowicz T, Ciuleanu TE, Radosavljevic D, Shacham-Shmueli E, Vrbanec D, Plate S, Mrsic-Krmpotic Z, Dank M, Purkalne G, Messinger D and Zielinski CC: FOLFOX4 plus cetuximab administered weekly or every second week in the first-line treatment of patients with KRAS wild-type metastatic colorectal cancer: A randomized phase II CECOG study. Ann Oncol 24: $1769-1777,2013$

20. Tabernero J, Ciardiello F, Rivera F, Rodriguez-Braun E, Ramos FJ, Martinelli E, Vega-Villegas ME, Roselló S, Liebscher S, Kisker O, et al: Cetuximab administered once every second week to patients with metastatic colorectal cancer: A two-part pharmacokinetic/pharmacodynamic phase I dose-escalation study. Ann Oncol 21: 1537-1545, 2010. 